\title{
Removal of Erythrocyte Membrane Iron In Vivo Ameliorates the Pathobiology of Murine Thalassemia
}

\author{
Paul V. Browne, ${ }^{\star}$ Oded Shalev, ${ }^{\star}$ Frans A. Kuypers, ${ }^{\ddagger}$ Carlo Brugnara, ${ }^{\S}$ Anna Solovey, ${ }^{\star}$ Narla Mohandas,, Stanley L. Schrier,,${ }^{\natural}$ \\ and Robert P. Hebbel ${ }^{*}$ \\ *Department of Medicine, University of Minnesota Medical School, Minneapolis, Minnesota 55455; $\doteqdot$ Oakland Children’s Hospital, \\ Oakland, California 94609; ${ }^{\S}$ Department of Pathology, Children's Hospital, Boston, Massachusetts 02115; "Lawrence Berkeley \\ Laboratory, Berkeley, California 94720; and "Department of Medicine, Stanford University Medical School, Stanford, California 94305
}

\begin{abstract}
Abnormal deposits of free iron are found on the cytoplasmic surface of red blood cell (RBC) membranes in $\beta$-thalassemia. To test the hypothesis that this is of importance to RBC pathobiology, we administered the iron chelator deferiprone (L1) intraperitoneally to $\beta$-thalassemic mice for $4 \mathrm{wk}$ and then studied RBC survival and membrane characteristics. L1 therapy decreased membrane free iron by $50 \%(P=$ 0.04) and concomitantly improved oxidation of membrane proteins $(P=0.007)$, the proportion of RBC gilded with immunoglobulin $(P=0.001), \mathrm{RBC}$ potassium content $(P<$ $0.001)$, and mean corpuscular volume $(P<0.001)$. Osmotic gradient ektacytometry confirmed a trend toward improvement of RBC hydration status. As determined by clearance of $\mathrm{RBC}$ biotinylated in vivo, $\mathrm{RBC}$ survival also was significantly improved in L1-treated mice compared with controls $(P=0.007)$. Thus, in vivo therapy with $\mathrm{L} 1$ removes pathologic free iron deposits from RBC membranes in murine thalassemia, and causes improvement in membrane function and RBC survival. This result provides in vivo confirmation that abnormal membrane free iron deposits contribute to the pathobiology of thalassemic RBC. (J. Clin. Invest. 1997. 100:1459-1464.) Key words: thalassemia • red blood cell • membrane $\bullet$ iron $\bullet$ chelation
\end{abstract}

\section{Introduction}

Accumulation of unpaired globin chains is a cardinal feature of the pathophysiology of thalassemia, and their precipitation and denaturation results in liberation of both heme and nonheme iron $(1,2)$. We previously demonstrated the presence of abnormal deposits of free iron (i.e., nonheme, nonferritin iron) on the cytoplasmic surface of thalassemic red blood cell (RBC) ${ }^{1}$ membranes (3). This pathologic redistribution of cellular iron

Address correspondence to Dr. Hebbel, Division of Hematology, Box 480 UMHC, Harvard St. at E. River Road, Minneapolis, MN 55455. Phone: 612-624-6104; FAX: 612-625-6919; E-mail: hebbe001@ maroon.tc.umn.edu

Received for publication 14 March 1997 and accepted in revised form 9 June 1997.

1. Abbreviations used in this paper: $\mathrm{DI}_{\max }$, deformability index; L1, deferiprone; $\mathrm{O}_{\text {hyp }}$, osmolality in the hypertonic range at which the deformability index has decreased to half its maximum value; $\mathrm{RBC}$, red blood cells.

J. Clin. Invest.

(C) The American Society for Clinical Investigation, Inc. 0021-9738/97/09/1459/06 \$2.00

Volume 100, Number 6, September 1997, 1459-1464

http://www.jci.org is believed to target injury to the RBC membrane, since iron is a catalyst in the formation of highly reactive oxygen intermediates such as hydroxyl radical. The consequent oxidation of membrane proteins and lipids causes loss of membrane function and, ultimately, contributes to premature cell destruction $(1,4,5)$. Similar free iron deposits (6) and hydroxyl radical generation (7) are hypothesized to underlie various membrane defects in sickle RBC as well (8). Thus, iron-mediated free radical chemistry taking place at the membrane/cytosolic interface is suggested to be a fundamental pathobiologic process.

Corroboration of the hypothesis that abnormal membrane iron deposition is of pathophysiologic significance in hemoglobinopathies could be obtained by removal of this iron. An opportunity to use this approach is provided by availability of the orally active chelator deferiprone (L1), long-term administration of which can induce negative iron balance in iron-overloaded patients (9). Unlike the traditional mainstay of chelation therapy deferoxamine, however, L1 can also readily permeate membranes (10). Indeed, L1 efficiently removes pathologic deposits of free iron from intact human thalassemic $\mathrm{RBC}$, both in vitro and in vivo (11). In the present investigation, we have tested the hypothesis that abnormal membrane iron deposits contribute to RBC membrane pathobiology by observing the beneficial effects of their L1-mediated removal in a murine model of $\beta$-thalassemia.

\section{Methods}

Sickle transgenic mice bred from a C57BL/6J background, heterozygous for linked human $\alpha$-globin and sickle $\beta$-globin transgenes, and homozygous for deletion of the murine $\beta$ major globin gene (12), were kindly provided by Dr. Mary Fabry (Albert Einstein College of Medicine, NY). Progeny of matings among these mice were screened using HPLC to characterize globin chain composition, and animals homozygous for the murine $\beta$-major globin deletion, but not containing the transgenes, were selected for establishment of a colony with murine $\beta$-thalassemia. Mice homozygous for this deletion have a thalassemia intermedia phenotype (13).

Thalassemic mice from multiple litters were randomly allocated to receive either L1 (kindly provided by Drs. Nancy Olivieri and Robert McClelland, University of Toronto) or saline vehicle as control. L1 was given intraperitoneally at a dose of $100 \mathrm{mg} / \mathrm{kg}$ twice daily for $6 \mathrm{~d}$ a wk, starting at age $1 \mathrm{mo}$. Saline vehicle was given on an identical schedule. The L1 and saline treatment groups were matched for sex and weight. The analysis of RBC survival commenced after 4 wk of therapy, which was continued during the survival study. After its completion, at $6 \mathrm{wk}$ of therapy, measurements of cellular parameters were conducted. Live animals were shipped to California so that ektacytometry and measurements of membrane thiol oxidation could be performed on fresh blood samples. Likewise, live animals were shipped to Boston for measurement of RBC indices and monovalent cation content. Because of the volumes of blood required for assessment of therapeutic efficacy, not all studies could be performed on all animals.

$R B C$ parameters. Red cell indicies were measured using a Tech- 
nicon-Bayer H3 automated analyzer (Bayer Diagnostics, Tarrytown, New York). Reticulocyte counts and hematocrits were measured manually. Red cell $\mathrm{Na}$ and $\mathrm{K}$ contents were determined after washing the cells five times in $172 \mathrm{mM}$ choline chloride, $1 \mathrm{mM} \mathrm{MgCl}_{2}$, and 10 $\mathrm{mM}$ tris-MOPS ( $\mathrm{pH} 7.40$ at $\left.4^{\circ} \mathrm{C}\right)$, as previously described (14).

Membrane iron. Exhaustively washed ghost membranes were prepared using iron-free buffers, as described previously (15). Heme iron (includes both free heme and hemoglobin) content was determined on membrane preparations dissolved in formic acid, as previously described (15). In separate membrane samples, presence of free iron (i.e., nonheme nonferritin iron) was defined according to the previously validated convention as reacting within 2 min with ferrozine in the presence of the denaturant SDS and the reducing agents ascorbate and sodium metabisulfite (6). Iron content was expressed in nanomoles per milligram of membrane protein, the latter determined by Bio-Rad (Richmond, CA) microprotein assay. Reagents used during membrane preparation and for the ferrozine assay were rendered iron-free by treatment with a chelating resin (Sigma Chemical Co., St. Louis, MO).

Membrane protein oxidation. Oxidation of membrane protein was assessed using thiol-disulfide exchange chromatography, exactly as described previously $(4,16)$. In brief, RBC membranes were prepared by hypotonic lysis with five washes in $20 \mathrm{vol}$ of cold, degassed sodium phosphate buffer ( $5 \mathrm{mmol} / \mathrm{liter}, \mathrm{pH} 8.0)$, containing $100 \mathrm{mg} / \mathrm{li}$ ter PMSF and $0.1 \mathrm{mmol} / \mathrm{L}$ EDTA. Aliquots solubilized in SDS were applied to the thiol-disulfide exchange column. Unbound protein, representing the oxidized thiol fraction, was expressed as a percentage of total protein applied to the column.

$R B C$ survival. Survival of RBC was measured using an established method (17). Mice were given three 1-mg injections of sterile NHS-X-Biotin (Calbiochem Corp., San Diego, CA) by tail vein over a 24-h period, which resulted in biotinylation of more than $95 \%$ of circulating RBC. Biotinylated RBC were detected by flow cytometry of samples labeled with FITC-conjugated streptavidin (Pierce Chemical Co., Rockford, IL). In brief, $2.5 \mu \mathrm{l}$ of capillary blood obtained by tail puncture was washed three times in PBS with $0.5 \%$ BSA (PBS/ BSA), and was resuspended in $1 \mathrm{ml}$ of PBS/BSA. Washed RBC (100$\mu \mathrm{l}$ aliquots) were incubated for $30 \mathrm{~min}$ at $4^{\circ} \mathrm{C}$ with $5 \mu \mathrm{l}$ of FITCstreptavidin. The samples were fixed in $2 \%$ paraformaldehyde/PBS after a further wash step, and were subsequently analyzed using a FACScan ${ }^{\circledR}$ (Becton Dickinson, Mountain View, CA) flow cytometer. Analytic gates were established using appropriate controls, including nonbiotinylated thalassemic RBC incubated with streptavidin-FITC and biotinylated RBC incubated with buffer. Labeled cells were expressed as a percentage of all cells counted (10,000 per sample).

Serial blood samples were collected during a 2-wk interval starting the day after completion of in vivo biotinylation (defined as day 1). L1 and saline injections were continued during this 2 -wk study period. Tail punctures performed during the survival analysis resulted in a cumulative blood loss of $\sim 50 \mu \mathrm{l}$, representing a loss of $<5 \%$ of total mouse blood volume over a 2 -wk interval. RBC survival was expressed as the time taken to reach $50 \%$ labeled cells (T1/2), calculated from the best-fit exponential curve.

$R B C$ ektacytometry. Intact RBC were studied using osmotic gradient ektacytometry, in which RBC deformation in response to shear stress is plotted as a continuous function of osmolality of the suspending medium, as described previously (18). For each sample, we recorded the degree of maximal RBC deformability, the deformability index $\left(\mathrm{DI}_{\max }\right)$, which provides a measure of RBC surface area. Also, the deformability index at isotonicity ( $290 \mathrm{mosmol} / \mathrm{kg}$ ) was determined $\left(\mathrm{DI}_{290}\right)$. The hypertonic region of the curves provided information on the state of $\mathrm{RBC}$ hydration, in the form of $\mathrm{O}_{\text {hyp }}$, the osmolality in the hypertonic range at which the deformability index has decreased to half its maximum value (18).

Membrane-bound immunoglobulin. Surface immunoglobulin was detected using a bead-rosette assay performed on RBC in suspension. Freshly-drawn tail blood $(10 \mu \mathrm{l})$ was washed twice in PBS/BSA, and resuspended to a final concentration of $5 \times 10^{6} \mathrm{RBC} / \mathrm{ml}$ in the same buffer. Polystyrene beads (Dynabeads M-450; Dynal Inc., Great Neck, NY) coated with affinity-purified goat anti-mouse IgG were washed four times in PBS/BSA and resuspended in wash buffer. Washed RBC $(50 \mu l)$ and washed antibody-coated beads $(10 \mu l)$ were mixed and incubated together at room temperature in round-bottom tubes for $1 \mathrm{~h}$, shaking gently. After gentle resuspension, $10 \mu \mathrm{l}$ of the mixture was placed on a glass slide under a coverslip. Using direct light microscropy, we determined the percentage of positive RBC with beads attached. At least $400 \mathrm{RBC}$ were counted in each experiment. Some experiments were performed in the presence of soluble mouse immunoglobulin (Sigma Chemical Co.). All experiments were performed with the microscopist blinded to the source of samples (L1- or saline-treated mice) to exclude observer bias.

Statistical methods. Means were compared using Student's $t$ test.

\section{Results}

Thalassemic phenotype. Hematologic parameters for both thalassemic and normal mice are presented in Table I. The values in our saline-treated thalassemic control group are consistent with a thalassemia intermedia phenotype, having elevated reticulocyte counts and microcytic anemia, as described previously (13). Additionally, the present studies of the salinetreated control group document that murine $\beta$-thalassemic RBC have abnormal amounts of membrane free iron, increased membrane thiol oxidation, diminished potassium content, increased amounts of surface Ig, abnormal ektacytometry profiles, and attenuated survival (Table I).

Membrane iron. As a consequence of in vivo L1 therapy, pathologic free iron measured on RBC membrane ghosts showed a significant reduction of $50 \%(P=0.04)$ in L1-treated thalassemic mice (Table I). Because L1 cannot chelate heme iron, predictably there was no difference in membrane-associated heme iron between L1-treated mice $(2.7 \pm 0.6 \mathrm{nmol} / \mathrm{mg}, n=$ 9) and saline-treated mice $(2.9 \pm 0.9 \mathrm{nmol} / \mathrm{mg}, n=10, P=$ $0.67)$.

Membrane protein oxidation. As a biochemical marker of iron-catalyzed membrane damage, membrane protein thiol oxidation was quantitated, and it was significantly decreased in L1-treated mice compared with saline-treated controls (Table I). The degree of oxidation seen in L1-treated mice approached that seen in normal mice.

RBC ektacytometry. Ektacytometry curves for groups of L1-treated and saline-treated thalassemic mice are shown in Fig. 1. The maximum value of the deformability index $\left(\mathrm{DI}_{\max }\right)$ in both of these thalassemic groups was virtually identical to that for normal mice (Table I), indicating no differences in RBC surface area. The deformability curve, however, was shifted to the left for the thalassemic mice, with the DI $\mathrm{max}_{\max }$ occurring at about $190 \mathrm{mosmol} / \mathrm{kg}$ (Fig. 1) as opposed to about $300 \mathrm{mosmol} / \mathrm{kg}$ for normal mice (not shown), indicating dehydrated red cells. L1 therapy resulted in a slight (not statistically significant) but consistent trend for the hypertonic component of the deformability curve to shift to the right (Fig. 1). This right shift, as documented by values for $\mathrm{O}_{\text {hyp }}$ (Table I), is consistent with improved RBC hydration in L1-treated animals (18), although it can reflect an improvement in membrane stiffness as well. This trend to improvement was further confirmed by the significant increase in deformability at isotonicity $\left(\mathrm{DI}_{290}\right)$, as shown in Table I.

Hematologic parameters. There was a small statistically significant decrease in hematocrit in the L1-treated group. Additionally, there was a significant increase in mean cell volume 


\begin{tabular}{|c|c|c|c|c|}
\hline & \multirow{2}{*}{$\begin{array}{c}\text { Normal reference } \\
\text { values }\end{array}$} & \multicolumn{3}{|c|}{ Thalassemic treatment groups } \\
\hline & & Saline-treated & L1-treated & $P$ value \\
\hline Membrane free iron $(\mathrm{nmol} / \mathrm{mg})$ & 0 & $4.4 \pm 2.3(8)$ & $2.2 \pm 1.8(10)$ & 0.04 \\
\hline Thiol oxidation $(\%)$ & $5.9 \pm 0.7$ & $18.7 \pm 3.4(3)$ & $7.8 \pm 1.5(3)$ & 0.007 \\
\hline $\mathrm{DI}_{\max }$ & $0.39 \pm 0.01$ & $0.40 \pm 0.02(6)$ & $0.41 \pm 0.01(6)$ & 0.3 \\
\hline $\mathrm{DI}_{290}$ & $0.39 \pm 0.01$ & $0.22 \pm 0.02(12)$ & $0.25 \pm 0.02(12)$ & 0.027 \\
\hline $\mathrm{O}_{\text {hyp }}(\mathrm{mosmol} / \mathrm{kg})$ & $438.3 \pm 10.9$ & $298.1 \pm 7.1(6)$ & $305.3 \pm 7.4(6)$ & 0.12 \\
\hline Hematocrit $(\%)$ & $43.7 \pm 1.5$ & $33.2 \pm 2.2(17)$ & $31.0 \pm 1.8(15)$ & 0.01 \\
\hline Reticulocyte count (\%) & $1.3 \pm 0.8$ & $26.5 \pm 6.2(17)$ & $23.7 \pm 5.3(15)$ & 0.18 \\
\hline Mean cell volume (fl) & $48.4 \pm 0.3$ & $34.1 \pm 1.2(10)$ & $45.6 \pm 4.8(10)$ & $<0.001$ \\
\hline $\operatorname{MCHC}(\mathrm{g} / \mathrm{dl})$ & $27.2 \pm 0.1$ & $27.6 \pm 0.4(10)$ & $25.6 \pm 0.9(10)$ & $<0.001$ \\
\hline $\mathrm{RBC} \mathrm{K}^{+}(\mathrm{mmol} / \mathrm{kg} \mathrm{Hb})$ & $500 \pm 24$ & $463.2 \pm 36.8(10)$ & $542 \pm 42.1(10)$ & $<0.001$ \\
\hline $\mathrm{RBC} \mathrm{Na}^{+}+\mathrm{K}^{+}(\mathrm{mmol} / \mathrm{kg} \mathrm{Hb})$ & $520 \pm 24$ & $484.4 \pm 38.2(10)$ & $562.1 \pm 43.2(10)$ & $<0.001$ \\
\hline Membrane Ig ( $\%$ positive cells) & $3.7 \pm 1.4$ & $10.1 \pm 2.4(16)$ & $6.3 \pm 3.3(14)$ & 0.001 \\
\hline RBC survival $\left(\mathrm{T}_{1 / 2}, \mathrm{~d}\right)$ & $20.0 \pm 1.2$ & $11.8 \pm 1.6(17)$ & $13.2 \pm 1.2(15)$ & 0.007 \\
\hline
\end{tabular}

Data shown as mean \pm SD (no. of animals tested). The illustrated $P$ values are for comparison of saline-treated versus L1-treated thalassemic animals. The illustrated normal reference values were obtained from C57BL/6J mice in the same laboratoies performing the measurements reported in this study.

and reduction in mean cell hemoglobin concentration in L1treated animals, both consistent with increased cell hydration (Table I). Reticulocyte count tended to be lower in L1-treated animals, although this difference was not statistically significant.

Cellular cation content. RBC contents of potassium ion alone and of total monovalent cations $\left(\mathrm{K}^{+}\right.$plus $\left.\mathrm{Na}^{+}\right)$were both significantly improved in animals given L1 compared to saline control (Table I), thus substantiating the suggestion of improved hydration obtained from ektacytometry and observation of changes in $\mathrm{RBC}$ indicies.

Membrane-bound immunoglobulin. The proportion of RBC carrying surface immunoglobulin detectable by the bead-

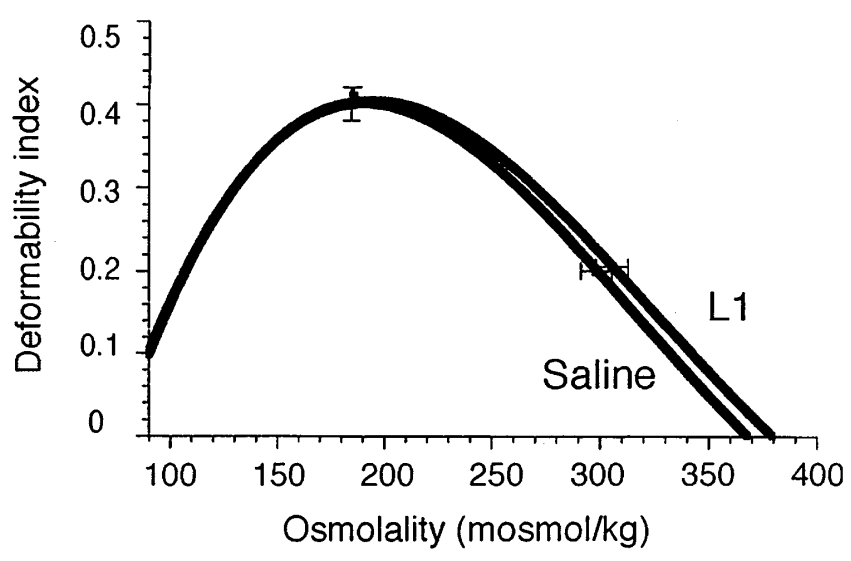

Figure 1. Ektacytometry curves show pooled data for $\mathrm{DI}_{\max }$ plotted against osmolality of suspending medium for L1- and saline-treated mice. Curves for the two groups are superimposed. Error bars $( \pm S D)$ are shown for the values of the $\mathrm{DI}_{\max }$ (vertical bars at the curve maximum) and $\mathrm{O}_{\text {hyp }}$ (horizontal bars in the hypertonic region). The latter reflects hydration status and shows a trend to be right-shifted for L1treated mice $(P=0.12, n=6)$. rosette antiimmunoglobulin assay significantly decreased in L1-treated mice (Table I). Addition of soluble immunoglobulin to the suspension medium reduced the proportion of $\mathrm{RBC}$-bead rosettes in saline-treated thalassemic mice to that observed in L1-treated mice, indicating that the RBC-bead interaction was immunoglobulin-mediated, and not occcurring via another mechanism (data not shown).

RBC survival. Survival half-time (T1/2) of RBC in normal C57BL/6J mice was $20.0 \pm 1.2 \mathrm{~d}(n=3)$. This value is consistent
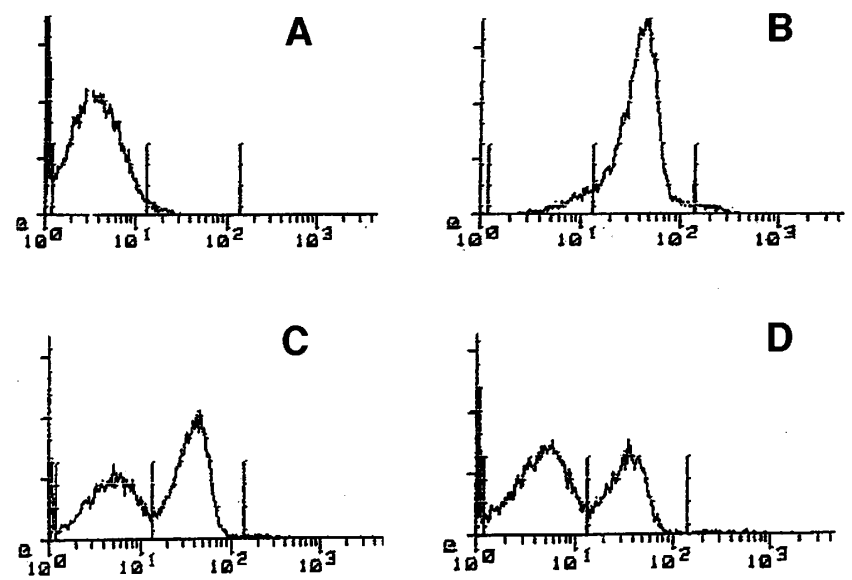

Figure 2. Representative fluorescence histograms illustrating monitoring for percentage of biotinylated cells are illustrated for a negative control obtained by streptavidin-FITC labeling of nonbiotinylated thalassemic $\mathrm{RBC}(A)$, and for serial blood samples taken on day $1(B)$, day $8(C)$, and day $13(D)$ after in vivo biotinylation of a salinetreated thalassemic mouse. Horizontal axis shows relative fluorescence intensity of FITC-labeled streptavidin bound to biotinylated RBC, expressed in arbitrary units. Vertical axis shows number of RBC (relative units). Markers indicate range of positively-labeled cells in each sample used for calculation of RBC survival. 


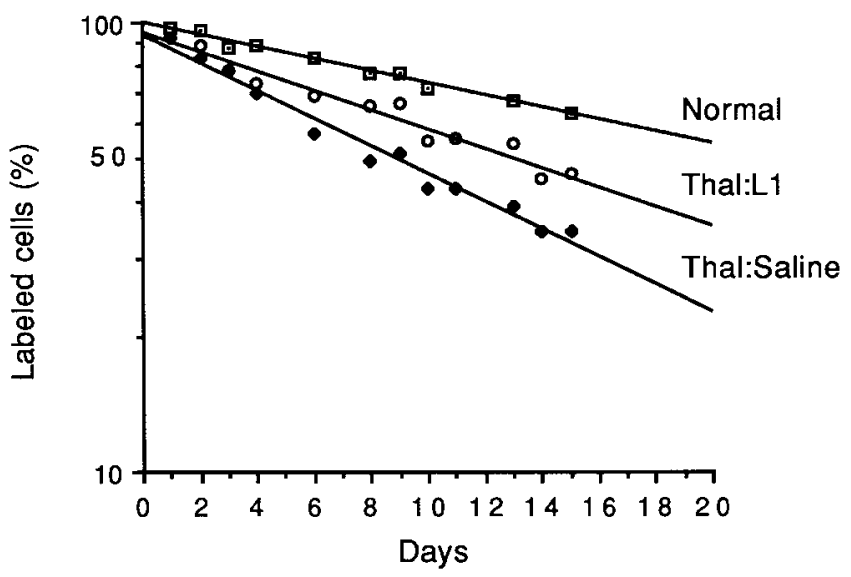

Figure 3. Representative RBC survival curves for individual normal (C57BL/6J) mice, and for L1- and saline-treated thalassemic mice. Data are plotted as percentage of labeled cells, on a logarithmic scale, against number of days.

with previous reports of murine RBC survival (17), confirming the validity of our RBC survival assay. Representative fluorescence histograms and survival plots for representative individual mice are shown in Figs. 2 and 3, respectively. For the entire group of animals (Fig. 4), the survival of RBC in L1-treated thalassemic mice $(\mathrm{T} 1 / 2=13.2 \pm 1.2 \mathrm{~d}, n=15)$ was significantly prolonged compared with survival of RBC in saline-treated control thalassemic mice $(\mathrm{T} 1 / 2=11.8 \pm 1.6 \mathrm{~d}, n=17, P=0.007)$.

\section{Discussion}

Using a murine model of $\beta$-thalassemia, we have tested the hypothesis, derived from a great variety of in vitro studies, that iron-catalyzed oxidative membrane damage contributes to the cellular pathobiology of hemoglobinopathic RBC. Insofar as

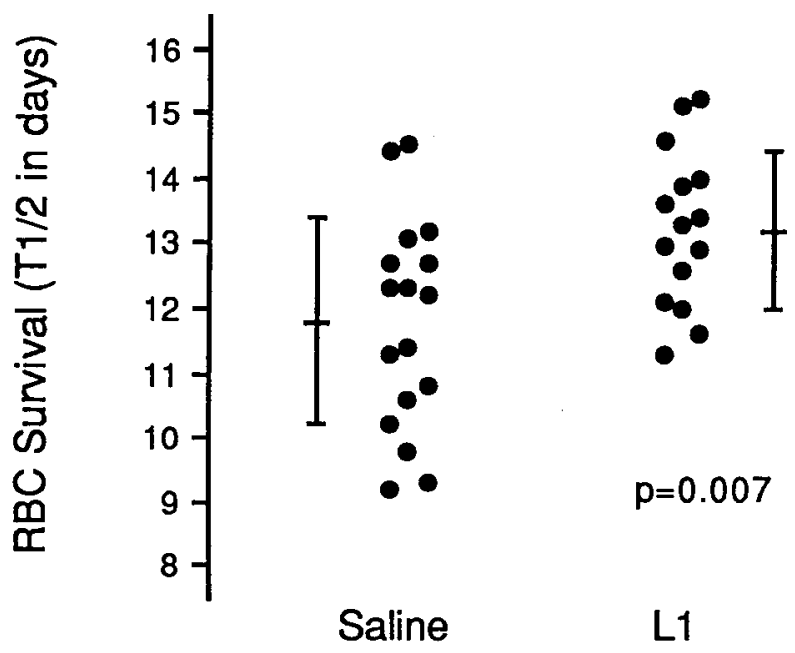

Figure 4. Distribution of individual RBC survivals in thalassemic mice, expressed as $\mathrm{T}_{1 / 2}$ (time in days to $50 \%$ biotinylated cells), are shown for L1 $(n=15)$ and saline $(n=17)$ treatment groups. this hypothesis is true, removal of pathologic membrane iron should be accompanied by improvement in membrane function. To accomplish this, we used the orally active chelator of free iron (but not of heme iron), L1, which we previously found to be able to penetrate RBC in vivo (11). Even though we found L1 to be only partially effective in removing abnormal RBC membrane iron in the used model of murine $\beta$-thalassemia, the level was sufficient to allow significant improvement in multiple measures of RBC membrane function (Table I) and, ultimately, in RBC survival (Fig. 4). Thus, these data provide the first evidence in an in vivo model that abnormal membrane free iron deposits indeed contribute to RBC membrane pathobiology and that chelation of such iron may improve RBC function.

To provide direct biochemical evidence for degree of oxidative membrane damage, we analyzed the status of membrane thiols using thiol-disulfide exchange chromatography. Although our present studies did not assess which specific membrane proteins were oxidized, they do indicate that in vivo chelation of RBC membrane free iron dramatically ameliorates the oxidation of membrane protein that is characteristic of human and murine thalassemic $\operatorname{RBC}(4,19-23)$. The remainder of our studies were directed at evaluation of selected cellular and membrane abnormalities. In this regard, the spectrum of features chosen for study here was dictated by our expectations regarding the oxidative pathobiology of thalassemia, as well as on the practical consideration of sampling requirements.

It appears that free iron removal did improve hydration state of RBC in L1-treated mice. This is indicated by the tendency towards a shift to the right in the hypertonic range of the deformability curves (although this could also result from improvement in membrane stiffness [23]), the significant change in $\mathrm{RBC}$ mean cell volume and mean cell hemoglobin concentration, and a convincing improvement in RBC potassium content. Cellular dehydration is a feature of $\beta$-thalassemia $(5,23)$, reflecting in part derangement of membrane transport functions caused by thiol oxidation (5), a defect potentially preventable with L1. Indeed, in humans with $\beta$-thalassemia, we have preliminarily observed improvement in $\mathrm{K}: \mathrm{Cl}$ cotransport in response to L1 therapy (24). In this study, however, we were unable to document any significant improvement in $\mathrm{K}: \mathrm{Cl}$ cotransport per se in these thalassemic mice (data not shown), so the component of RBC dehydration that is iron-induced in this model apparently is related to other ion-handling pathways.

The reduction in surface immunoglobulin we observed in L1-treated thalassemic mice is also of interest. We tested for this because of the reported relationship between surface Ig and coclusters of band 3 and oxidatively denatured hemoglobin that are implicated in attenuation of RBC survival in human thalassemia (25). Moreover, we have demonstrated recently in vitro that membrane-associated pathologic free iron can directly catalyze formation of hemichrome (26), and we have observed in thalassemic humans in vivo a modest reduction in membrane hemichrome during L1 therapy (11). While we observed L1 therapy to cause a significant improvement in gilding of the RBC membrane with Ig, we were unable to discern any L1-induced diminution of membrane hemichrome in these animals. It is likely that this is because instability of unpaired globin chains is of paramount importance in deposition of hemichrome on murine thalassemic RBC membranes. The mechanism by which free iron independently promotes immu- 
noglobulin deposition, however, is unknown. It is conceivable that this is related to band 3 aggregation resulting from iron binding to the potential thiol cluster formed by the cytoplasmic sequence of band 3 molecules (27), although this has not been tested experimentally.

As an overall measure of the impact of L1 chelation on oxidative $\mathrm{RBC}$ pathobiology in murine $\beta$-thalassemia, the observed improvement of RBC lifespan in L1-treated mice is particularly important. Although the magnitude of this L1mediated benefit appears modest, it should be noted that we were here attempting to demonstrate an alteration in $\mathrm{RBC}$ kinetics under the adverse circumstance of having the rather high background level of random RBC destruction characteristic of mice (17). Accordingly, in this setting, and with the only partial removal of membrane iron effected by L1, the demonstration of any significant improvement in RBC survival is very encouraging. Our study was not designed to assess the precise reason for improvement of RBC survival, which could lie in improved hydration, reduced immunoglobulin gilding, diminished membrane instability, or perhaps in other factors.

It is possible that more complete chelation of RBC pathologic free iron, and/or concomitant reduction of membranedenatured hemoglobin, might have been associated with an even better outcome. The dose of L1 we selected was already high enough to manifest its myelosuppressive effect (28), as evidenced by the observed reduction of hematocrit and reticulocyte count. In any case, the goal of the present study was not to improve the anemia of murine thalassemia, but rather to provide one test of the hypothesis that abnormal deposits of membrane free iron contribute to the oxidative pathobiology of hemoglobinopathic RBC membranes. In fact, insofar as the beneficial effect of free iron chelation is exerted on mechanisms for peripheral RBC destruction, we would predict that this approach would be even more beneficial in sickle disease than in thalassemia. Sickle RBC manifest the same membrane defects studied here (29), but are not influenced by the unstable unpaired globin chains that are of paramount importance to thalassemic pathobiology. Even though we have focused on the effects of chelation of iron in mature RBC, in the thalassemic setting it is possible that chelation of abnormally distributed iron in erythroid precursors may be even more important. Much of the pathology of thalassemia is attributable to the gross ineffective erythropoiesis characteristic of this disorder. Premature destruction of erythroid precursors within the bone marrow is likely to reflect, at least in part, cellular injury associated with excess iron. Further study is required to address the role of pathologic free iron deposition, and possible benefits of iron chelation, in thalassemic erythropoiesis.

We speculate that chelation of erythroid iron with chelating agents capable of penetrating $\mathrm{RBC}$ in vivo may improve human thalassemic pathobiology sufficiently to diminish peripheral, and possibly intramedullary, hemolysis. A reduction in hemolytic rate, particularly if associated with improved erythroid marrow function, would diminish transfusion requirement, and ultimately the hemosiderosis that accounts for much of the mortality of thalassemia. Removal of abnormal erythroid iron deposits represents a novel and potentially important objective for clinical chelation therapy in thalassemia. It may be, however, that accomplishing this removal will require development of membrane-permeable chelators able to remove membrane iron without exerting concomitant myelosuppressive effects.

\section{Acknowledgments}

We thank Drs. Stephana Choong and Kuan Sheng for excellent technical assistance.

This work was supported by a Fellowship of the Cooley's Anemia Foundation (to P.V. Browne) and by the National Institutes of Health: DK32094 and HL27059 to F.A. Kuypers, HL15157 to C. Brugnara, HL31579 to N. Mohandas, DK32094 to S.L. Schrier, and HL37528 and HL55552 to R.P. Hebbel.

\section{References}

1. Shinar, E., and E.A. Rachmilewitz. 1990. Oxidative denaturation of red blood cells in thalassemia. Semin. Hematol. 27:70-82.

2. Scott, M.D., J.J.M. van den Berg, T. Repka, P. Rouyer-Fessard, R.P. Hebbel, Y. Beuzard, and B.H. Lubin. 1993. Effect of excess $\alpha$-hemoglobin chains on cellular and membrane oxidation in model $\beta$-thalassemic erythrocytes. J. Clin. Invest. 91:1706-1712.

3. Repka, T., O. Shalev, R. Reddy, J. Yuan, A. Abrahamov, E.A. Rachmilewitz, P.S. Low, and R.P. Hebbel. 1993. Nonrandom association of free iron with membranes of sickle and $\beta$-thalassemic erythrocytes. Blood. 82:3204-3210.

4. Advani, R., E. Rubin, N. Mohandas, and S.L. Schrier. 1992. Oxidative red blood cell membrane injury in the pathophysiology of severe mouse $\beta$-thalassemia. Blood. 79:1064-1067.

5. Olivieri, O., L. De Franceschi, M.D. Capellini, D. Girelli, R. Corrocher, and C. Brugnara. 1994. Oxidative damage and erythrocyte membrane transport abnormalities in thalassemias. Blood. 84:315-320.

6. Kuross, S.A., and R.P. Hebbel. 1988. Nonheme iron in sickle erythrocyte membranes: Association with phospholipids and potential role in lipid peroxidation. Blood. 72:1278-1285.

7. Repka, T., and R.P. Hebbel. 1991. Hydroxyl radical formation by sickle erythrocyte membranes: role of pathologic iron deposits and cytoplasmic reducing agents. Blood. 78:2753-2758.

8. Hebbel, R.P. 1990. The sickle erythrocyte in double jeopardy: autoxidation and iron decompartmentalization. Semin. Hematol. 27:51-69.

9. Olivieri, N.F., G.M. Brittenham, D. Matsui, M. Berkovitch, L.M. Blendis, R.G. Cameron, R.A. McClelland, P.P. Liu, D.M. Templeton, and G. Koren. 1995. Iron-chelation therapy with oral deferiprone in patients with thalassemia major. N. Engl. J. Med. 332:918-922.

10. Kontoghiorghes, G.J. 1988. Structure/red blood cell permeability, activity of iron(III) chelator complexes. Inorganica Chimica Acta. 151:101-106.

11. Shalev, O., T. Repka, A. Goldfarb, L. Grinberg, A. Abrahamov, N.F. Olivieri, E.A. Rachmilewitz, and R.P. Hebbel. 1995. Deferiprone (L1) chelates pathologic iron deposits from membranes of intact thalassemic and sickle red blood cells both in vitro and in vivo. Blood. 86:2008-2013.

12. Fabry, M., R.L. Nagel, A. Pachnis, S.M. Suzuka, and F. Costantini. 1992. High expression of human $\beta^{\mathrm{S}}$ and $\alpha$-globins in transgenic mice: hemoglobin composition and hematological consequences. Proc. Natl. Acad. Sci. USA. 89: 12150-12154.

13. Skow, L.C., B.A. Burkhart, F.M. Johnson, R.A. Popp, D.M. Popp, S.Z. Goldberg, W.F. Anderson, L.B. Barnett, and S.E. Lewis. 1983. A mouse model for beta-thalassemia. Cell. 34:1043-1052.

14. Armsby, C.C., C. Brugnara, and S.L. Alper. 1995. Cation transport in mouse erythrocytes: Role of $\mathrm{K}+/ \mathrm{Cl}-$ cotransport in regulatory volume decrease. Am. J. Physiol (Cell Physiol. 37) 268:C894-C902.

15. Kuross, S., B.H. Rank, and R.P. Hebbel. 1988. Excess heme in sickle erythrocyte inside-out membranes: Possible role in thiol oxidation. Blood. 71: 876-882.

16. Rank, B., J. Carlsson, and R.P. Hebbel. 1985. Abnormal redox status of membrane-protein thiols in sickle erythrocytes. J. Clin. Invest. 75:1531-1537.

17. Hoffman-Fezer, G., J. Mysliwietz, W. Mörtbauer, H.J. Zeitler, E. Eberle, U. Hönle, and S. Thierfelder. 1993. Biotin labeling as an alternative nonradioactive approach to determination of red cell survival. Ann. Hematol. 67:81-87.

18. Clark, M.R., N. Mohandas, and S. Shohet. 1983. Osmotic gradient ektacytometry: comprehensive characterization of red cell volume and surface maintenance. Blood. 61:899-910.

19. Shinar, E., E.A. Rachmilewitz, and S.E. Lux. 1989. Differing erythrocyte membrane skeletal protein defects in alpha and beta thalassemia. J. Clin. Invest. 83:404-410.

20. Rouyer-Fessard, P., M.-C. Garel, C. Domenget, D. Guetarni, D. Bachir, P. Colonna, and Y. Beuzard. 1989. A study of membrane protein defects and $\alpha$ hemoglobin chains of red blood cells in human $\beta$ thalassemia. J. Biol. Chem. 264:19092-19098.

21. Rouyer-Fessard, P., K. Leroy-Viard, C. Domenget, A. Mrad, and Y. Beuzard. 1990. Mouse $\beta$ thalassemia, a model for the membrane defects of erythrocytes in the human disease. J. Biol. Chem. 265:20247-20251.

22. Advani, R., S. Sorenson, E. Shinar, W. Lande, E. Rachmilewitz, and S.L. Schrier. 1992. Characterization and comparison of the red blood cell mem- 
brane damage in severe human $\alpha$ - and $\beta$-thalassemia. Blood. 79:1058-1063.

23. Schrier, S., E. Rachmilewitz, and N. Mohandas. 1989. Cellular and membrane properties of alpha and beta thalassemic erythrocytes are different: implication for differences in clinical manifestations. Blood. 74:2194-2202.

24. De Franceschi, L., O. Shalev, A. Piga, M. Collell, O. Olivieri, R. Corrocher, R.P. Hebbel, N. Olivieri, and C. Brugnara. 1996. Deferiprone (L1) treatment removes erythrocyte membrane free iron and affects $\mathrm{K}-\mathrm{Cl}$ cotransport activity in homozygous $\beta$ thalassemia. Blood. 88 (Suppl.):24b.

25. Yuan, J., R. Kannan, E. Shinar, E.A. Rachmilewitz, and P.S. Low. 1992. Isolation, characterization, and immunoprecipitation studies of immune com- plexes from membranes of $\beta$-thalassemic erythrocytes. Blood. 79:3007-3013.

26. Shalev, O., and R.P. Hebbel. 1996. Catalysis of soluble hemoglobin oxidation by free iron on sickle red cell membranes. Blood. 87:3948-3952.

27. Thevenin, B.J.-M., B.M. Willardson, and P.S. Low. 1989. The redox state of cysteines 201 and 317 of the erythrocyte anion exchanger is critical for ankyrin binding. J. Biol. Chem. 264:15886-15892.

28. Olivieri, N. 1996. Long-term therapy with deferiprone. Acta Haematologica. 95:37-48

29. Hebbel, R.P. 1991. Beyond hemoglobin polymerization: the red blood cell membrane and sickle disease pathophysiology. Blood. 77:214-237. 